Preoperative mapping of the eloquent cortical areas using navigated transcranial magnetic stimulation combined with intraoperative neuronavigation for intracerebral lesions 


\title{
Preoperative mapping of the eloquent cortical areas using navigated transcranial magnetic stimulation combined with intraoperative neuronavigation for intracerebral lesions
}

\author{
G. Petrescu ${ }^{1,2}$, Cristina Gorgan ${ }^{3}$, A. Giovani ${ }^{1}$, F.M. Brehar ${ }^{1,4}$, \\ R.M. Gorgan ${ }^{1,4}$ \\ ${ }^{1}$ Neurosurgery Department, “Bagdasar-Arseni” Emergency Hospital, Bucharest, ROMANIA \\ ${ }^{2} \mathrm{PhD}$ student, "Carol Davila” University of Medicine and Pharmacy, ROMANIA \\ ${ }^{3}$ Neurology Department, “Bagdasar-Arseni” Emergency Hospital, Bucharest, ROMANIA \\ ${ }^{4}$ Neurosurgery Department, "Carol Davila" University of Medicine and Pharmacy, ROMANIA
}

\begin{abstract}
Introduction: Maximal surgical resection with the preservation of cortical functions is the treatment of choice for brain tumors. Achieving these objectives is especially difficult when the tumor is located in an eloquent area. Navigated transcranial magnetic stimulation (nTMS) is a modern non-invasive, preoperative method for defining motor and speech eloquent areas. Material and methods: Patients with tumors located in motor and speech eloquent areas who presented at our institution between March 2017 and December 2017 were prospectively included. Exclusion criteria were frequent generalized epileptic seizures and cranial implants. For lesions involving motor eloquent areas we performed a nTMS motor mapping and for lesions involving speech eloquent areas we supplemented the motor mapping with speech and language mapping. MR images were exported from the nTMS system in a DICOM format and then loaded in the intraoperative neuronavigation system. Based on these findings, the optimal entry point and trajectory were determined, in order to achieve a maximum surgical resection of the lesion, while avoiding new postoperative neurological deficits. Results: Nineteen patients underwent an nTMS brain mapping procedure between March 2017 and December 2017. In all cases a motor mapping procedure was done, but only in eight cases a speech mapping was also performed. Three patients presented new minor postoperatory deficits that consecutively remitted. The rest of the patients presented no added neurological deficits after surgery. In five cases the preexistent deficit was ameliorated after surgery and in three cases the deficit remitted. In one patient there was no improvement in the neurologic deficit after surgery. Conclusion: nTMS is a reliable
\end{abstract}


tool for the preoperative planning of eloquent area lesions. It must be taken into account that functional areas have a high individual variability. Therefore, knowing preoperatively the extent of the eloquent area helps the neurosurgeon adapt the surgical approach in order to obtain a better functional outcome.

Key words: Navigated transcranial magnetic stimulation, Preoperative mapping, Neuronavigation, Eloquent areas, Intracerebral lesions

\section{Introduction}

Maximal surgical resection with the preservation of cortical functions is the treatment of choice for brain tumors (1-3). Achieving these objectives is especially difficult when the tumor is located in an eloquent area (i.e. precentral gyrus, postcentral gyrus, Broca's area, Wernicke's area). Numerous methods have been used in order to determine preoperative and/or intraoperative the eloquent areas. Navigated transcranial magnetic stimulation (nTMS) is a modern non-invasive, preoperative method for defining motor and speech eloquent areas $(4,5)$. This method integrates a frameless neuronavigation system and a TMS coil that delivers a precise focal electrical field at the level of the cortex.

This article describes our experience with nTMS brain mapping and reviews the advantages and common pitfalls of this method.

\section{Material and methods}

\section{Patients}

Patients with tumors located in motor and speech eloquent areas who presented at our institution between March 2017 and December 2017 were prospectively included. Exclusion criteria were frequent generalized epileptic seizures and cranial implants. A thorough neurologic examination was performed pre- and post-operatory for each patient.

\section{Brain mapping procedure}

The brain mapping procedure was performed using a Nexstim Navigated Brain Stimulation System 5 (Nexstim, Helsinki, Finland). A high-quality head MRI with 1-mm thick slices, that included the vertex, nasion and ears was performed for all patients and then was loaded in the nTMS system. These anatomical landmarks are used for registering the patient into the frameless stereotactic navigation. Consecutively, surface electromyography (EMG) recording electrodes (Neuroline 720, AMBU, Ballerup, Denmark) are placed at the level of abductor pollicis brevis (APB), abductor digiti minimi (ABM), flexor carpi radialis (FCR) and when needed also at the level of the tibilias anterior (TA), abductor hallucis (AH) and extensor hallucis longus (EHL). A figure-of-eight stimulation coil is used to deliver precise stimulation pulses based on the neuronavigation guidance. After the initial determination of the motor threshold, the actual mapping is performed. TMS pulses were delivered in a systematic way at $110 \%$ of the resting motor threshold, in the areas of interest. Motor-evoked potentials (MEPs) were recorded by the EMG and their exact location was noted on the MRI. Additionally, for lesions involving speech eloquent areas we 
supplemented the motor mapping with speech and language mapping. Using the NexSpeech Software (Nexstim, Helsinki, Finland) the patients were subjected to an object-naming task by displaying illustrations of common objects or numbers on a monitor and their performance was recorded by a video camera (fig. 1). In order to determine the baseline, the initial task was performed without TMS. The picture display time (PDT) and the interpicture interval (IPI) were usually $700 \mathrm{~ms}$ and $2500 \mathrm{~ms}$ respectively, with variations based on the patients' grade of neurologic deficit. Pictures that were not recognized by the patients were excluded. This procedure was typically repeated two or three times. Then, navigated repetitive TMS (nrTMS) pulses were delivered as a train of 5 pulses with a frequency of $5 \mathrm{~Hz}$, at $100 \%$ of the resting motor threshold and at a picture-to-trigger (PTT) interval of $0 \mathrm{~ms}$. The results were then analyzed and compared to the baseline. Naming errors were defined as follows: no response, semantic error, performance error, error due to muscle stimulation and other. Afterwards, the results were recorded on the brain MRI.

\section{Neuronavigation system integration and surgical planning}

MR images were exported from the nTMS system in a digital imaging and communications in medicine (DICOM) format and then loaded in the intraoperative neuronavigation system SonoWand Invite (SonoWand AS, Trondheim, Norway). Based on these findings, the optimal entry point and trajectory were determined, in order to achieve a maximum surgical resection of the lesion, while avoiding new postoperative neurological deficits. In cases of infiltrative tumors, an intraoperative 3D ultrasound probe was used to determine the extent of resection.

\section{Results}

Nineteen patients underwent an nTMS brain mapping procedure between March 2017 and December 2017. There were 13 male patients and 6 female patients. The median (range) age of the patients was 53 (18-73) years. In all cases a motor mapping procedure was done, but only in eight cases a speech mapping was also performed. In seventeen cases a surgical resection of the lesion was performed, while one patient underwent only a stereotactic biopsy procedure. In one case the surgery was contraindicated because of the late-stage of primary cancer and other serious comorbidities. The pathology report revealed eight cases of glioblastoma, one case of a grade II astrocytoma, three cases of cavernoma, one of meningioma, one case of non-Hodgkin lymphoma and one cerebral abscess. Other three cases were cerebral metastasis of various primary origin. The patient that did not undergo surgery had a history of pulmonary carcinoma that relapsed, so the cerebral lesion probably had a pulmonary origin. Three patients presented new minor post-operatory deficits that consecutively remitted. The rest of the patients presented no added neurological deficits after surgery. In five cases the preexistent deficit was ameliorated after surgery and in three cases the deficit remitted. In one patient there was no improvement in the neurologic deficit after surgery. No seizures occurred during the procedures and no other adverse effects were noted. The characteristics of each case are described in Table 1. 
TABEL 1

Depicts the characteristics of each case. $\mathrm{M}$ - male, F - female, GBM- glioblastoma, NHL - nonHodgkin Lymphoma, F - frontal, P - parietal, T - temporal, LV - lateral ventricle, Th- thalamus, L-

left, $\mathrm{R}$ - right, ? - not verified

\begin{tabular}{|c|c|c|c|c|c|c|c|c|}
\hline No. & Sex & Age & Lesion & Location & Mapping & Procedure & Preop. deficits & Postop. deficits \\
\hline 1 & $\mathrm{M}$ & 61 & GBM & FTP/R & Motor & Surgery & None & None \\
\hline 2 & M & 37 & Abscess & $\mathrm{F} / \mathrm{L}$ & Motor & Surgery & R. Hemiparesis & Remitting \\
\hline 3 & M & 73 & GBM & $\mathrm{FTP} / \mathrm{L}$ & Motor & Surgery & None & None \\
\hline 4 & M & 53 & Metastasis & $\mathrm{FP} / \mathrm{L}$ & Motor & Surgery & R. Hemiparesis & $\begin{array}{l}\text { Remitting; } \\
\text { Facial palsy - remitted }\end{array}$ \\
\hline 5 & $\mathrm{~F}$ & 23 & Cavernoma & $\mathrm{FP} / \mathrm{L}$ & Motor & Surgery & R. brachial paresis & Remitted \\
\hline 6 & M & 21 & Cavernoma & $\mathrm{P} / \mathrm{R}$ & Motor & Surgery & None & $\begin{array}{l}\text { R. crural deficit - } \\
\text { remitting }\end{array}$ \\
\hline 7 & M & 18 & Cavernoma & $\mathrm{F} / \mathrm{L}$ & Motor/Language & Surgery & None & None \\
\hline 8 & $\mathrm{~F}$ & 70 & Metastasis & $\mathrm{FP} / \mathrm{L}$ & Motor/Language & Surgery & None & None \\
\hline 9 & M & 65 & GBM & $\mathrm{TP} / \mathrm{L}$ & Motor/Language & Surgery & $\begin{array}{l}\text { Aphasia, } \\
\text { R. Hypoesthesia }\end{array}$ & Remitting \\
\hline 10 & $\mathrm{M}$ & 62 & GBM & $\mathrm{T} / \mathrm{L}$ & Motor/Language & Surgery & $\begin{array}{l}\text { R. Hemiparesis } \\
\text { Dysphasia }\end{array}$ & $\begin{array}{l}\text { Remitting } \\
\text { Remitted }\end{array}$ \\
\hline 11 & M & 73 & Metastasis? & $\mathrm{F} / \mathrm{R}$ & Motor & None & L. hemiparesis & - \\
\hline 12 & M & 41 & NHL & $\mathrm{LV} / \mathrm{L}$ & Motor & Surgery & $\begin{array}{l}\text { Aphasia } \\
\text { R. hemiparesis }\end{array}$ & $\begin{array}{l}\text { Aphasia } \\
\text { R. hemiparesis }\end{array}$ \\
\hline 13 & M & 46 & GBM & $\mathrm{FP} / \mathrm{L}$ & Motor/Language & Surgery & $\begin{array}{l}\text { R. facial palsy } \\
\text { R. hemiparesis }\end{array}$ & Remitting \\
\hline 14 & $\mathrm{~F}$ & 49 & Meningioma & $\mathrm{FP} / \mathrm{L}$ & Motor & Surgery & None & $\begin{array}{l}\text { R. crural hemiparesis - } \\
\text { remitting }\end{array}$ \\
\hline 15 & $\mathrm{~F}$ & 31 & Astrocytoma gr. II & $\mathrm{Th} / \mathrm{L}$ & Motor & Biopsy & R. hemiparesis & R. hemiparesis \\
\hline 16 & M & 54 & GBM & $\mathrm{F} / \mathrm{L}$ & Motor/Language & Surgery & $\begin{array}{l}\text { Aphasia } \\
\text { R. hemiparesis }\end{array}$ & Remitted \\
\hline 17 & $\mathrm{~F}$ & 64 & Metastasis & $\mathrm{FP} / \mathrm{R}$ & Motor/Language & Surgery & L. Hemiparesis & Remitting \\
\hline 18 & $\mathrm{~F}$ & 61 & GBM & $\mathrm{T} / \mathrm{L}$ & Motor/Language & Surgery & $\begin{array}{l}\text { Aphasia } \\
\text { Confusion }\end{array}$ & Remitted \\
\hline 19 & M & 42 & GBM & $\mathrm{TP} / \mathrm{L}$ & Motor & Surgery & None & None \\
\hline
\end{tabular}

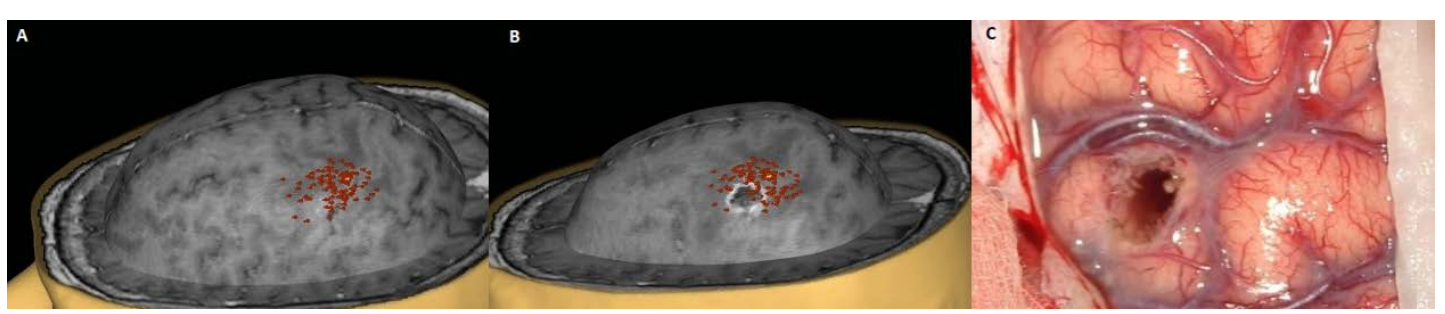

Figure 2 - A patient with a squamous cell carcinoma metastasis located at the level of the left central sulcus. A red dots represent low-intensity MEPs and the yellow dots medium-intensity MEPs in relation to the cortical surface. B - red dots represent low-intensity MEPs and the yellow dots medium-intensity MEPs in relation to the tumour. C - intraoperative image after the tumor was completely resected; The small corticomy and the numerous drainage veins at the level of the cortex and their structural integrity following surgery must be noted.

Figure available in color online only 


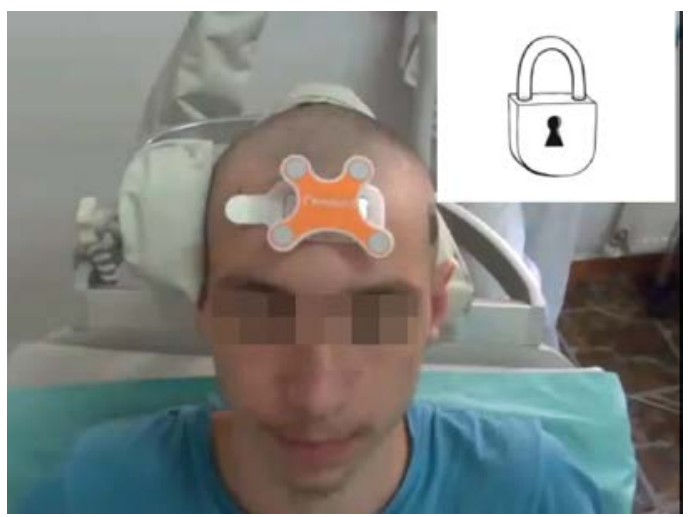

Figure 1 - Snapshot from the video recorded during the nTMS language mapping. In the upper right corner is the representation of a lock that was displayed on the monitor during the object-naming task. Figure available in color online only

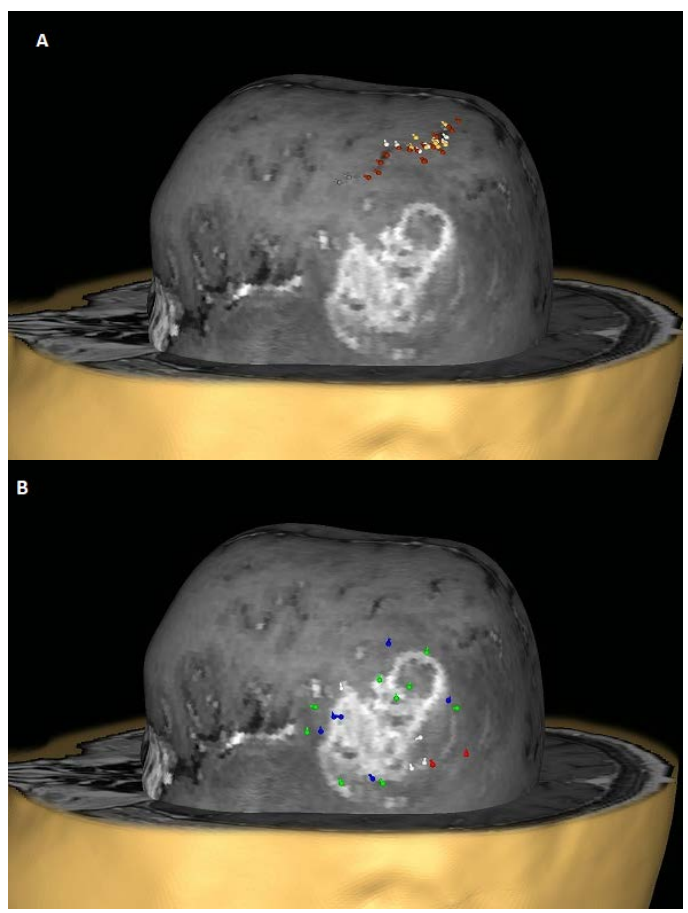

Figure 3 - Left temporoparietal glioblastoma in a 65year-old patient that presented with aphasia and right-sided hypoesthesia. A - motor mapping, red dots represent low-intensity MEPs, yellow dots medium-intensity MEPs and white dots high- intensity MEPs; B- language mapping, white dots no response error, green dots - performance error, blue dots - semantic error, red dots - unclear interpretation. Figure available in color online only

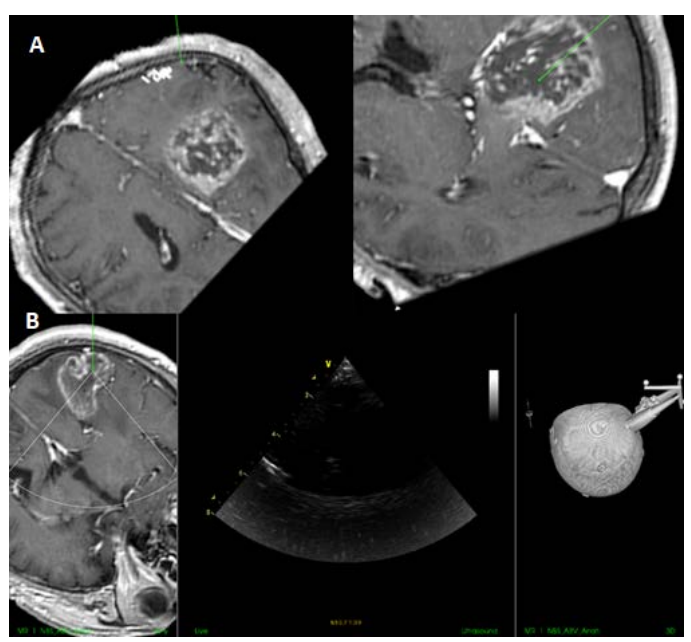

Figure 4 - Intraoperative view from the neuronavigation system. A - nTMS-positive points appear as white dots. $\mathrm{B}-$ the $3 \mathrm{D}$ ultrasound probe is used to determine in real-time the extent of resection.

Figure available in color online only

\section{Discussion}

The theory that the brain is structured in multiple static centers, each responsible for a certain cortical function is more and more challenged by recent advances in imaging and mapping techniques and as well by the clinical experience that proves that patients with lesions in presumed eloquent areas can still recover function (4). A hodotopic model is proposed that defines the brain as a set of large dynamic networks that cooperate in order to preserve or recover the cortical functions $(6,7)$. Brain tumors tend to disrupt the normal anatomy and can even determine plastic restructuring $(8,9)$. Taking these into account, 
when facing an eloquent area lesion, neurosurgeons cannot rely only on good anatomical knowledge. The surgical planning must also include brain mapping methods that can help determine precise location of the eloquent area and its relation to the lesion.

Many methods have been used for mapping of cortical eloquent areas, preoperatively, as well as intraoperatively, including: functional MRI (fMRI), diffusion tensor imaging (DTI), magnetoencephalography (MEG), positron emission tomography (PET), single-photon emission computed tomography (SPECT) and direct cortical stimulation (DCS) (10-12). fMRI has been regarded as one of the most frequently used and reliable methods for preoperative mapping (13-15), but more recent studies question its' precision and role, particularly in tumors that affect the normal vascularization and consequently alter the metabolic mapping $(16,17)$. Regarding motor mapping DCS remains the "gold-standard" method for assessing the precise location of the motor function (5). nTMS motor mapping has been proved to have comparable results to DCS $(17,18)$. Also, nTMS has a better accuracy than fMRI (19), while also having the advantage of not being limited by the usage of voluntary motor tasks that do not necessarily trigger only the primary motor areas and can also be used in patients that are less compliant (20).

Another usage of nTMS is that the precise location of MEPs can be used as seed-points when performing DTI, thus achieving a better and more accurate image of the corticospinal tract (21-23). Knowledge of such information is critical for obtaining a good surgical outcome, since damage to the subcortical structures can probably produce more severe and permanent neurological deficits than an injury localized at the cortical surface (4).

In our series, a 53-year old male presented with a left frontoparietal lesions, located at the level of the central sulcus. The preoperative neurologic exam revealed right-sided hemiparesis. During the nTMS mapping procedure, only low-intensity and a couple medium-intensity MEPs were recorded (fig. 2A). This could be explained by the preexistence of a motor deficit. A few MEPs were recorded above the tumor (fig. 2B). Given the numerous drainage veins located at the cortical surface, in the vicinity of the tumor, only one entry-point could be chosen, in order to preserve the integrity of the veins, hence avoiding a poor outcome. Following the resection of the tumor through an area with nTMS-positive motor points, the patient had a transient worsening of the neurologic deficit. This situation is not uncommon. Moser et al. published a study in which of the thirteen patients that underwent the resection of nTMS-positive motor points, eight had new permanent neurologic deficits and two only transient ones (24).

Similarly, to the motor mapping, the DCS with an awake patient is considered the "goldstandard" for language mapping (25). In order to obtain the best results it is necessary to achieve a state of optimal conscious sedation (10). Besides the technical difficulty, not all patients are comfortable enough to undergo an awake surgery. In this context, nTMS offers the extraordinary advantage of doing the 
procedure preoperatory, offering a comfortable solution for both the patient and the surgeon. The only question that remains is how reliable is nTMS for language mapping.

Language mapping using nTMS was proven more sensitive, but less specific than fMRI, when compared with DCS, but similar results to DCS can be achieved by combining nTMS and fMRI $(26,27)$. Picht et al. reported that even though, nTMS was less specific than DCS, particularly in the supramarginal, angular and posterior superior temporal gyri, the negative responses were similar to those recorded by DCS (28). Because of the repetitive nature of the pulses used for language mapping, side effects including seizures and headaches might be encountered. In our series no such events were record, this being consistent with data reported from other study (14).

In our series, a 65-year-old male, that presented with aphasia and right-sided hypoesthesia was admitted. The brain MRI revealed a left temporoparietal mass (fig 3). The optimal entry point was chosen accordingly to the location of the languagepositive points and post-operatory the patient's neurologic function improved.

In some cases, it is very difficult to perform and interpret a language mapping because of the preexistent neurological deficits. Two patients had difficulties in recognizing the objects that were illustrated in the pictures, the problem being especially in the initiation of the command, because after the examiner aided them with the first syllabus of the word, their performance improved. In these two cases, the patients did very well when they were asked to recognize numbers, so this baseline was used instead. Schwarzer et al. also noted that in patients with aphasia it is more difficult to rely on the nTMS language mapping (29).

When combined with intraoperative neuronavigation, nTMS offers a major benefit when, as it highlights the eloquent areas which are sometimes much more extended than expected and helps the neurosurgeon choose the safest entry points. Also, the 3D ultrasound probe adds more value, the surgical team being able to determine in real-time the extent of resection (fig.4). Combined, these tools facilitate the achievement of a maximum resection while avoiding new postoperative neurological deficits.

\section{Conclusions}

Navigated transcranial magnetic stimulation is a reliable tool for the preoperative planning of eloquent area lesions. Its results are similar to DCS for motor mapping and it does not have the disadvantages of awake surgery regarding language mapping. It must be taken into account that functional areas have a high individual variability. Therefore, knowing preoperatively the extent of the eloquent area helps the neurosurgeon adapt the surgical approach in order to obtain a better functional outcome.

\section{Correspondence}

Petrescu George Emil Dragoș

gedpetrescu@gmail.com 


\section{References}

1. Lee C-H, Kim DG, Kim JW, Han JH, Kim YH, Park C$\mathrm{K}$, et al. The role of surgical resection in the management of brain metastasis: a 17-year longitudinal study. Acta Neurochir (Wien). 2013;155(3):389-97.

2. Sanai N, Berger MS. G Lioma E Xtent of R Esection and and Methods. Neurosurgery. 2008;62(4):753-66.

3. Li YM, Suki D, Hess K, Sawaya R. The influence of maximum safe resection of glioblastoma on survival in 1229 patients: Can we do better than gross-total resection? J Neurosurg. 2016;124(4):977-88.

4. Picht T. Current and potential utility of transcranial magnetic stimulation in the diagnostics before brain tumor surgery. CNS Oncol. 2014;3(4):299-310.

5. Lefaucheur JP, Picht T. The value of preoperative functional cortical mapping using navigated TMS. Neurophysiol Clin. 2016;46(2):125-33.

6. De Benedictis A, Duffau H. Brain hodotopy: From esoteric concept to practical surgical applications. Neurosurgery. 2011;68(6):1709-23.

7. Duffau H. Hodotopy, neuroplasticity and diffuse gliomas. Neurochirurgie. 2017;63(3):259-65.

8. Takahashi S, Jussen D, Vajkoczy P, Picht T. Plastic relocation of motor cortex in a patient with LGG (low grade glioma) confirmed by NBS (navigated brain stimulation). Acta Neurochir (Wien). 2012 Nov;154(11):2003-8; discussion 2008.

9. Conway N, Wildschuetz N, Moser T, Bulubas L, Sollmann N, Tanigawa N, et al. Cortical plasticity of motor-eloquent areas measured by navigated transcranial magnetic stimulation in patients with glioma. J Neurosurg. 2017;127(November):1-11.

10. Darder JMG, Lopez PG. Management of Brain Tumors in Eloquent Areas. In: Diagnostic Techniques and Surgical Management of Brain Tumors. InTech; 2011. p. 15-22.

11. Mazziotta JC. Imaging: window on the brain. Arch Neurol. 2000 Oct;57(10):1413-21.

12. Desmond JE, Sum JM, Wagner AD, Demb JB, Shear PK, Glover GH, et al. Functional MRI measurement of language Lateralization in Wada-tested patients. Brain. 1995;118(6):1411-9.

13. Lehéricy S, Duffau H, Cornu P, Capelle L, Pidoux B, Carpentier A, et al. Correspondence between functional magnetic resonance imaging somatotopy and individual brain anatomy of the central region: comparison with intraoperative stimulation in patients with brain tumors. J Neurosurg.

14. Picht T, Schmidt S, Woitzik J, Suess O. Navigated brain stimulation for preoperative cortical mapping in paretic patients: Case report of a hemiplegic patient. Neurosurgery. 2011;68(5):1475-80.

15. Tieleman A, Deblaere K, Van Roost D, Van Damme $\mathrm{O}$, Achten E. Preoperative fMRI in tumour surgery. Eur Radiol. 2009;19(10):2523-34.

16. Holodny AI, Schulder M, Liu WC, Wolko J, Maldjian JA, Kalnin AJ. The effect of brain tumors on BOLD functional MR imaging activation in the adjacent motor cortex: Implications for image-guided neurosurgery. Am J Neuroradiol. 2000;21(8):1415-22.

17. Krieg SM, Shiban E, Buchmann N, Gempt J, Foerschler A, Meyer B, et al. Utility of presurgical navigated transcranial magnetic brain stimulation for the resection of tumors in eloquent motor areas. J Neurosurg. 2012;116(5):994-1001.

18. Picht T, Schmidt S, Brandt S, Frey D, Hannula H, Neuvonen T, et al. Preoperative functional mapping for rolandic brain tumor surgery: Comparison of navigated transcranial magnetic stimulation to direct cortical stimulation. Neurosurgery. 2011;69(3):581-8.

19. Coburger J, Musahl C, Henkes H, Horvath-Rizea D, Bittl M, Weissbach C, et al. Comparison of navigated transcranial magnetic stimulation and functional magnetic resonance imaging for preoperative mapping in rolandic tumor surgery. Neurosurg Rev. 2013 Jan;36(1):65-6.

20. Frey D, Schilt S, Strack V, Zdunczyk A, Sler JR, Niraula B, et al. Navigated transcranial magnetic stimulation improves the treatment outcome in patients with brain tumors in motor eloquent locations. Neuro Oncol. 2014;16(10):1365-72.

21. Frey D, Strack V, Wiener E, Jussen D, Vajkoczy P, Picht T. A new approach for corticospinal tract reconstruction based on navigated transcranial stimulation and standardized fractional anisotropy values. Neuroimage. 2012;62(3):1600-9.

22. Krieg SM, Buchmann NH, Gempt J, Shiban E, Meyer $B$, Ringel F. Diffusion tensor imaging fiber tracking using navigated brain stimulation-a feasibility study. Acta Neurochir (Wien). 2012;154(3):555-63.

23. Weiss Lucas C, Tursunova I, Neuschmelting V, Nettekoven C, Oros-Peusquens AM, Stoffels G, et al. Functional MRI vs. navigated TMS to optimize M1 seed 
volume delineation for DTI tractography. A prospective study in patients with brain tumours adjacent to the corticospinal tract. NeuroImage Clin. 2017;13:297-309.

24. Moser T, Bulubas L, Sabih J, Conway N, Wildschutz $\mathrm{N}$, Sollmann N, et al. Resection of Navigated Transcranial Magnetic Stimulation-Positive Prerolandic Motor Areas Causes Permanent Impairment of Motor Function. Neurosurgery. 2017;81(1):99-110.

25. Duffau H, Lopes M, Arthuis F, Bitar A, Sichez J-P, Van Effenterre R, et al. Contribution of intraoperative electrical stimulations in surgery of low grade gliomas: a comparative study between two series without (1985-96) and with (1996-2003) functional mapping in the same institution. J Neurol Neurosurg \&amp;amp; Psychiatry. 2005 Jun 1;76(6):845 LP-851.

26. Ille S, Sollmann N, Hauck T, Maurer S, Tanigawa N, Obermueller $\mathrm{T}$, et al. Impairment of preoperative language mapping by lesion location: a functional magnetic resonance imaging, navigated transcranial magnetic stimulation, and direct cortical stimulation study. J Neurosurg. 2015 Aug;123(2):314-24.

27. Ille S, Sollmann N, Hauck T, Maurer S, Tanigawa N, Obermueller $\mathrm{T}$, et al. Combined noninvasive language mapping by navigated transcranial magnetic stimulation and functional MRI and its comparison with direct cortical stimulation. J Neurosurg. 2015 Jul;123(1):212-25. 28. Picht T, Krieg SM, Sollmann N, Rösler J, Niraula B, Neuvonen $\mathrm{T}$, et al. A comparison of language mapping by preoperative navigated transcranial magnetic stimulation and direct cortical stimulation during awake surgery. Neurosurgery. 2013;72(5):808-19.

29. Schwarzer V. Aphasia and cognitive impairment decrease the reliability of rnTMS language mapping. 2017; 